\title{
Fatigue and Buckling Strength Analysis of the Wing Hinge in the Creo Simulation Environment
}

\author{
Karol Semrád \\ Department of Aviation Technical Studies, Faculty of Aeronautics, Technical University Kosice, Kosice 04121, Slovak Republic
}

Received: January 10, 2013 / Accepted: February 7, 2013 / Published: April 25, 2013.

\begin{abstract}
This paper provides background information on fatigue and describes the methodology used in Creo Simulation (Pro/Mechanica) fatigue analysis. It covers history and physics of fatigue, the E-N approach — Strain Cycles Includes the effect of mean residual stresses, hysteresis loop capture and rainflow cycle counting. Factors that affect fatigue life include component size, loading type, surface finish, surface treatment and effect of surface treatments on endurance limit. This paper describes practical problem solution and fatigue strength analysis of the wing-hinge to fuselage connection in Creo Simulation (Pro/Mechanica) environment. It also includes a procedure for calculating the buckling load factor and failure index.
\end{abstract}

Key words: Fatigue, Creo Simulation, Pro/Mechanica, S-N diagram, E-N diagram, rainflow cycle counting, Wöhler line, CAE (computer aided engineering), CAD (computer aided design), log life, confidence of life, failure index, BLF (buckling load factor).

\section{Introduction}

The majority of component designs involve parts subjected to fluctuating or cyclic loads. Such loading induces fluctuating or cyclic stresses that often result in failure by fatigue. About $95 \%$ of all structural failures occur through fatigue mechanism. Between 1852 and 1870, August Wöhler, a German railway engineer, set up and conducted the first systematic fatigue investigation. His data were plotted, in terms of nominal stress (S) vs. number of cycles to failure $(\mathrm{N})$, what has become known as the S-N diagram (Wöhler line) [1].

\section{History and Physics of Fatigue}

The physical development of a crack is generally divided into two separate stages, bearing relation to the phase of crack initiation phase and crack growth. Fatigue cracks are initiated through the release of shear strain energy. Following the 2nd stage, the crack propagates perpendicularly to the direction of the applied load. As the physical mechanism for fatigue is

Corresponding author: Karol Semrád, Ph.D.-Ing., research field: applied mechanics. E-mail: karol.semrad@tuke.sk. divided into two stages, the methods of analysis are also divided into two stages. The 1st stage is a typically analysis based on the local strain (E-N) approach, while the 2nd stage is an analysis based on the fracture mechanics approach. A complete fatigue prediction could therefore combine both methods: Total Life $=$ Life to initiation + Life taken to propagate crack to failure.

Many aerospace applications use flexible components made of very ductile materials. In such cases, cracks propagate relatively slowly, so the fracture mechanics approaches are considered more appropriately.

The $\mathrm{S}-\mathrm{N}$ analysis is unsuitable for performing CAE analysis on components containing local areas of plasticity because it uses elastic stress as the input and fatigue initiation is driven by local plastic strain. For this reason, methods based on local strain (E-N) are more universally suitably. To this product, the E-N approach has been applied [1].

\section{The E-N Approach}

The E-N approach uses fatigue tests, subjected to various types of cyclic loading, such as small-scale bending, torsion, tension and compression to measure 
fatigue life. The results are plotted in terms of strain (E) vs. cycles to failure $(\mathrm{N})$ on an E-N diagram.

Owing to variable amplitudes of loading, it is necessary to extract the fatigue damaging cycles from the signal before starting the evaluation of the damage inflicted by each cycle. The total damage is the sum of the damage caused by each individual cycle. Each fatigue cycle is extracted by a process known as the hysteresis loop capture.

Creo Simulation uses a generic set of fatigue properties to model low alloy steels, unalloyed steels, aluminium alloys and titanium alloys. A very efficient algorithm has been developed to perform cycle extraction, known as rainflow cycle counting. It is the algorithm that Creo Simulation uses (Fig. 1).

When a stress-strain hysteresis loop is closed, then the strain range and mean stress are reverted and the damage calculated using the E-N curve modified for mean stress correction. The analysis was carried out over the whole strain time signal until all the cycles have been extracted and the total damage evaluated.

Creo Simulation usually uses a linear elastic solution to determine the pseudo-elastic strains in a component. The solution ignores the plasticity. Before proceeding with the fatigue analysis, these strains are automatically converted into non-linear elastic-plastic strains using Neuber's relationship.

There are some factors that affect fatigue life (component size, loading type, surface finish). For example, if an axial tension load produces a strain $\varepsilon$, the strain produced under a bending load would be $1.25 \varepsilon$. If machining reduces the endurance limit by $30 \%$, then cold rolling will recover the loss by increasing the limit to $70 \%$ [2].

\section{Problem Solution by Creo Simulation}

We are designing a wing-to-fuselage connection. One of the components, where analysis is required, is the wing hinge (Fig. 2).

Wing hinges have never been known to fail trough fatigue in the past but we are now required to prove this before the component progresses to the next stage in
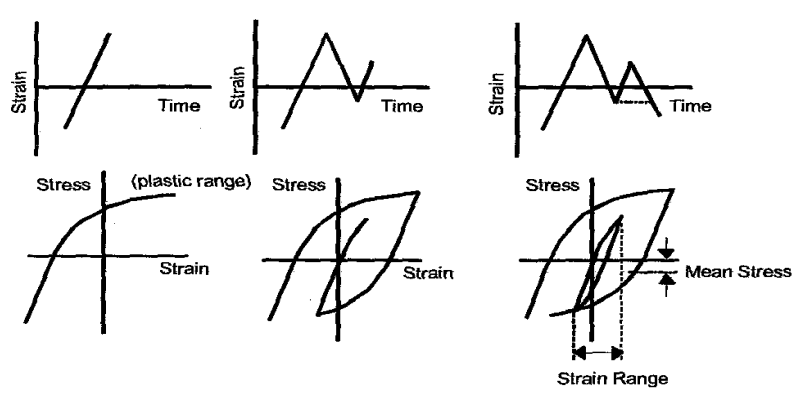

Strain Amplitude $=1 / 2$ Strain Range

Fig. 1 Rainflow cycle counting algorithm.
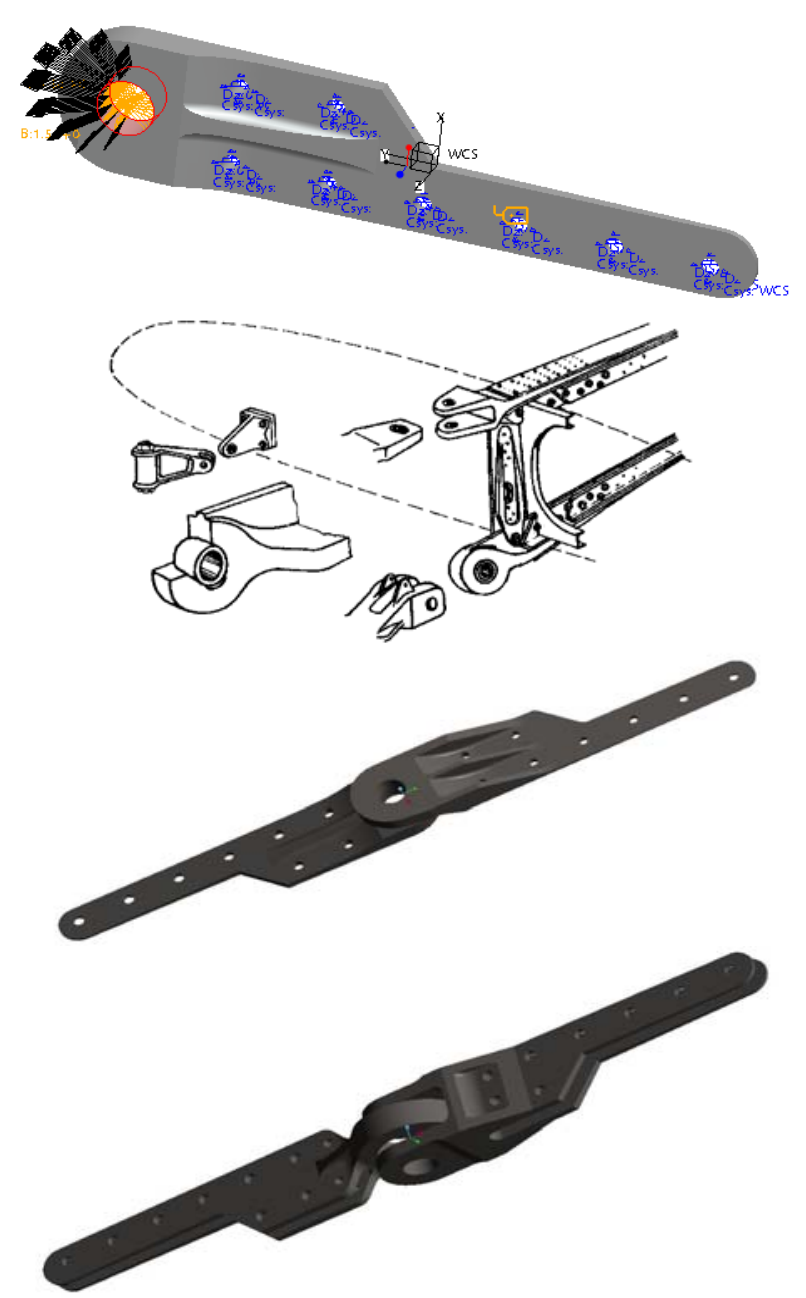

Fig. 2 CAD models of the wing hinges.

the design. To our estimates, the wing hinge has a maximum tensile force of $15000 \mathrm{~N}$ applied to the connecting hinge eye (Fig. 2). The material chosen is steel (For details see Fig. 3). The new wing hinge has a target life of approximately 10 million cycles under a peak-to-peak loading (Fig. 3). The results of both the static and fatigue analyses are presented in Figs. 4-6. 


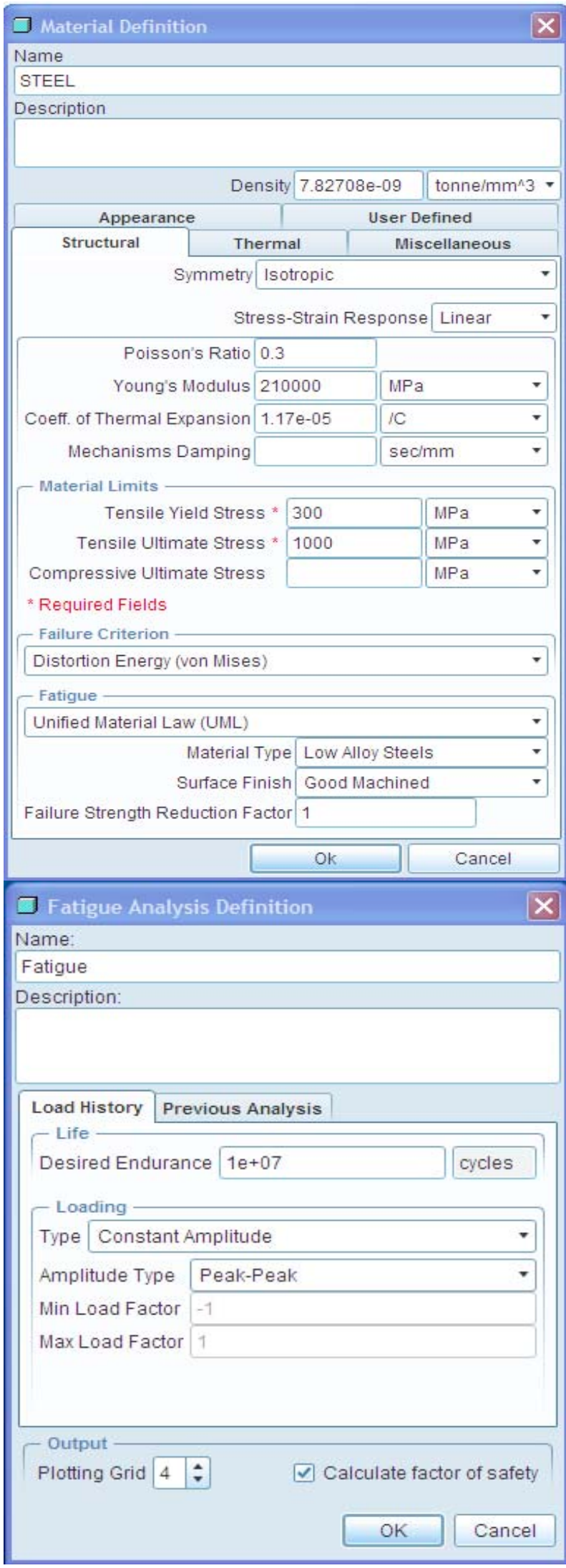

Fig. 3 Material property form and fatigue analysis form.

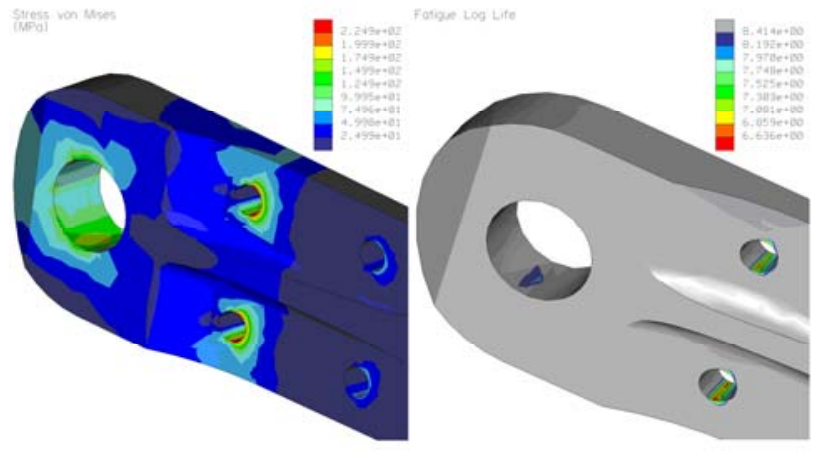

Fig. 4 Stress von Mises and fatigue log-life plots.

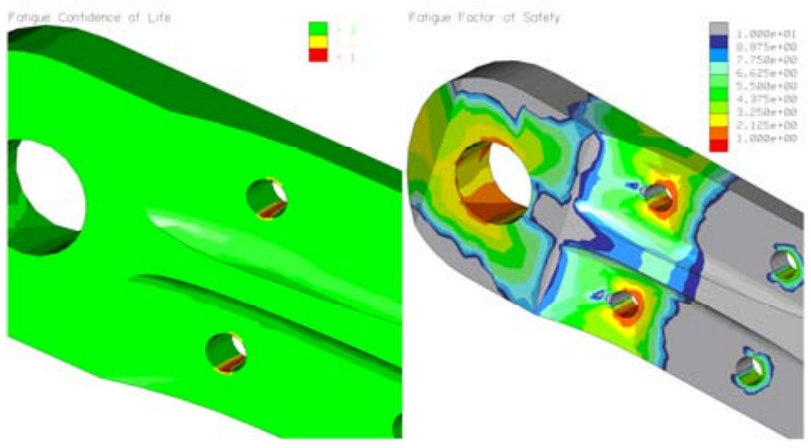

Fig. 5 Fatigue confidence of life and fatigue factor of safety plots.

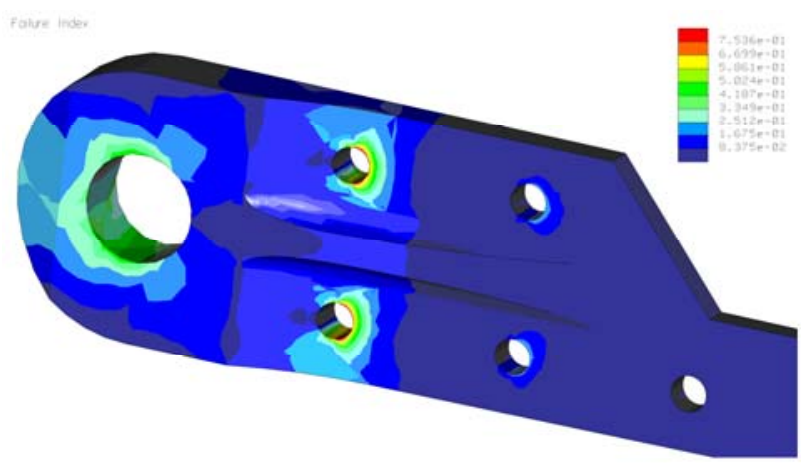

Fig. 6 Failure index plot.

\section{Conclusions}

Look at the results from the static stress and log life plots (Fig. 4). Notice where the fatigue cracks are likely to form. The minimum log life is 6.636 that means $10^{6.636}$ or 4325138 cycles at a point where the Stress von Mises has its maximum of $225 \mathrm{MPa}$. Have a look at the other plots (Fig. 5). Notice how the confidence of life is all green. The minimum factor of safety suggests a permissible overload before fatigue life is jeopardized. 
Creo Simulation does not analyze plastic behavior. That is something the user must check for. When the model stress exceeds the material yield stress, it has permanently deformed and will not return to its original shape. Creo Simulation treats the stress-strain curve as if it is linear even beyond the yield stress point. The way we check to see if the material is plastically deformed is to see if the calculated stress levels exceed the material Yield Stress. This means that we must be aware of the yield stress, or yield strength, of the material we are using. Although strictly speaking, material does not fail in the plastic region. Creo Simulation considers the plastic region to be failure. Creo Simulation's Failure Strength is the level of stress at which the material starts to plastically deform. We select a failure determination method, then enter the cutoff stress limit for that method (Fig. 3) [2].

Creo Simulation results allow you to plot a failure index measure with a color fringe plot (Fig. 6). The calculated stresses are compared to the cutoff stresses and an index is plotted. The index is interpreted as follows: Less than 1-Material has not yielded. In this Case, failure index is 0.7 max. Equal to or greater than 1-Material has yielded. The color legend can be adjusted so that failure indexes greater than 1 are colored red. This way we can look at the result window for the color red. Red would indicate that under the applied load the material has failed, or that the calculated stress has exceeded our limit [3].

The results of a previously run static analysis are the starting point for a buckling analysis. Creo Simulation buckling analysis is a linear eigenvalue bifurcation instability analysis. It will check to see if the model will buckle under a given compressive loading condition. The BLF (Buckling Load Factor) is the magnification factor by which the loads applied in a previous specified static analysis would have to be multiplied to produce the critical buckling load: BLF less than 1 -Model has buckled. BLF greater than 1-Model has not buckled. In this case, BLF $=103$ for maximum compressive force of wing hinge $15000 \mathrm{~N}$ [4].

Creo Simulation buckling analysis will typically overestimate the buckling load in comparison to real world tests. This means the Creo Simulation buckling load is somewhat high, and that the part may buckle below the buckling load that Creo Simulation predicts. We should divide the Creo Simulation BLF by a factor of 2-3 in order to provide a conservative solution. For stress results, we should use the static analysis results. For stress results at the area of buckling, multiply the stresses from the static analysis (Fig. 4) with the buckling load factor. The buckling load factor can be used as a limit in Creo Simulation optimization studies [4].

\section{References}

[1] J.E. Shingley, Ch.R. Mischke, R.G. Budynas, Mechanical Engineering Design (in Czech), VUT v Brně, VUTIUM, 2010.

[2] Pro/ENGINEER Wildfire 3 Help Page, Cambridge University Engineering Department, Cambridge UK, http://www.eng.cam.ac.uk/DesignOffice/cad/.

[3] L. Novotný, Using finite element method for calculation of some crack characteristic, in: Winter Workshop of Applied Mechanics, Faculty of Mechanical Engineering, CTU in Prague, 2006, pp. 24-25.

[4] CADTRAIN-COACH for Pro/ENGINEER, COACH e-Learning Solutions, Irvine USA, http://www.cadtrain.com/. 